\title{
The GRB 030329 host: a blue low metallicity subluminous galaxy with intense star formation ${ }^{\star}$
}

\author{
J. Gorosabel ${ }^{1}$, D. Pérez-Ramírez ${ }^{2}$, J. Sollerman ${ }^{3}$, A. de Ugarte Postigo ${ }^{1}$, J. P. U. Fynbo ${ }^{4}$, A. J. Castro-Tirado ${ }^{1}$, \\ P. Jakobsson ${ }^{4,5}$, L. Christensen ${ }^{6}$, J. Hjorth ${ }^{4}$, G. Jóhannesson ${ }^{5}$, S. Guziy ${ }^{1}$, J. M. Castro Cerón ${ }^{4}$, G. Björnsson ${ }^{5}$, \\ V. V. Sokolov ${ }^{7}$, T. A. Fatkhullin ${ }^{7}$, and K. Nilsson ${ }^{4,8}$
}

1 Instituto de Astrofísica de Andalucía (IAA-CSIC), PO Box 03004, 18080 Granada, Spain

e-mail: [jgu;ajct; deugarte;gss]@iaa.es

2 Universidad de Jaén, Departamento de Física (EPS), Virgen de la Cabeza 2, Jaén 23071, Spain

e-mail: dperez@ujaen.es

3 Stockholm Observatory, Department of Astronomy, AlbaNova, 10691 Stockholm, Sweden e-mail: jesper@astro.su.se

4 Niels Bohr Institute, University of Copenhagen, Juliane Maries Vej 30, 2100 Copenhagen $\emptyset$, Denmark e-mail: [jfynbo;pallja; jens;kim]@astro.ku.dk; josemari@alumni.nd.edu

5 Science Institute, University of Iceland, Dunhaga 3, 107 Reykjavík, Iceland

e-mail: [gudlaugu;gulli]@raunvis.hi.is

6 Astrophysikalisches Institut, 14482 Potsdam, Germany e-mail: 1christensen@aip.de

7 Special Astrophysical Observatory of the Russian Academy of Sciences, Nizhnij Arkhyz, 357147 Karachai-Cherkessia, Rusia e-mail: [sokolov; timur]@sao.ru

8 European Southern Observatory, Karl-Schwarzschild-Strasse 2, 85748 Garching bei Munchen, Germany

Received 25 January 2005 / Accepted 20 July 2005

\section{ABSTRACT}

We present broad band photometry and spectroscopic observations of the host galaxy of GRB 030329. Analysis of the spectral emission lines shows that the host is likely a low metallicity galaxy $(Z \sim 0.004)$. The spectral energy distribution (SED) constructed with the photometric points has been fitted using synthetic and observational templates. The best SED fit is obtained with a starburst template with an age of $\sim 150 \mathrm{Myr}$ and an extinction $A_{\mathrm{v}} \sim 0.6$. We find that the GRB 030329 host galaxy is a subluminous galaxy $\left(L \sim 0.016 L^{\star}\right)$ with a stellar mass of $\gtrsim 10^{8} M_{\odot}$. Three independent diagnostics, based on the restframe UV continuum, the [O II], and the Balmer emission lines, provide a consistent unextinguished star formation rate of $\sim 0.6 M_{\odot} \mathrm{yr}^{-1}$, implying a high unextinguished specific star formation $\operatorname{rate}\left(\sim 34 M_{\odot} \mathrm{yr}^{-1}\left(L / L^{\star}\right)^{-1}\right)$. We estimate that the unextinguished specific star formation rate of the GRB 030329 host is higher than $\sim 93.5 \%$ of the galaxies at a similar redshift.

Key words. gamma rays: bursts - Galaxy: fundamental parameters - techniques: photometric - techniques: spectroscopic

\section{Introduction}

An extremely bright gamma-ray burst (GRB) was detected on 2003 March 29 at 11:37:14.67 UT (GRB 030329; Vanderspek et al. 2003) by the French Gamma Ray Telescope (FREGATE),

\footnotetext{
* Based on observations made with the Nordic Optical Telescope, operated on the island of La Palma jointly by Denmark, Finland, Iceland, Norway, and Sweden, in the Spanish Observatorio del Roque de los Muchachos of the Instituto de Astrofísica de Canarias. Based on data taken at the 2.2-m and $3.5-\mathrm{m}$ telescopes of the Centro Astronómico Hispano Alemán de Calar Alto, operated by the Max Planck institute of Heidelberg and Centro Superior de Investigaciones Científicas. The spectral observations were obtained at the European Southern Observatory, Cerro Paranal (Chile), under the Director's Discretionary Time programme 271.D-5006(A).
}

the Wide Field X-ray Monitor (WXM), and the Soft X-ray Camera (SXC) instruments aboard the High Energy Transient Explorer II (HETE-2) spacecraft (Ricker et al. 2003). With a duration of approximately $30 \mathrm{~s}$ in the $30-400 \mathrm{keV}$ energy range, GRB 030329 falls into the "long-duration" category of GRBs (Kouveliotou et al. 1993).

Before the HETE-2 era, two main scenarios were favoured to explain the nature of long GRBs. One of them suggested that GRBs occur when two compact objects (such as black holes or neutron stars) merge (Eichler et al. 1989; Narayan et al. 1992). This would imply that GRBs occur after massive star formation has ended in a galaxy, since the time-scale for binary objects to merge is long ( $\gtrsim 1 \mathrm{Gyr}$ ). A second scenario, the so-called collapsar scenario, suggested that GRBs are associated with the core-collapse of massive stars (supernovae or 
hypernovae: Woosley 1993; Paczyński 1998; MacFadyen \& Woosley 1999). This scenario predicts a supernova (SN) accompanying the GRB, which thus must be coincident with the epoch of star formation in the host.

The studies carried out in 2003 for GRB 030329 (Hjorth et al. 2003; Stanek et al. 2003; Sokolov et al. 2003; Kawabata et al. 2003) strongly confirmed previous evidences (Bloom et al. 1999; Castro-Tirado \& Gorosabel 1999) that long GRBs are physically linked to supernova explosions. The origin of the short GRBs is still unknown, so here we will discuss only the hosts of long GRBs. Radio, optical and/or infrared afterglows have been observed for $\sim 70$ long GRBs, and the majority of these coincide with star forming galaxies.

The pioneering statistical work applying the spectral energy distribution (SED) fitting technique to GRB host galaxies was published by Sokolov et al. (2001), who argued that GRB hosts correspond to starburst galaxies. A number of other complementary studies have been carried out in the past, studying the properties of different GRB host samples (Chary et al. 2002; Berger et al. 2003; Le Floc'h et al. 2003; Tanvir et al. 2004) or centred on a particular host galaxy (Djorgovski et al. 2001a, 2003; Hjorth et al. 2002). Recently, a statistical analysis by Christensen et al. (2004a; hereafter C04A) based on the SED of a sample of 10 GRB host galaxies indicated the similarity of GRB hosts and the young starburst galaxies present in the Hubble Deep Field (HDF; Williams et al. 2000).

A good knowledge of the host SED makes it possible to derive the key parameters to determine the conditions that favour a GRB event, e.g., the star formation rate (SFR), the dominant stellar population age, the stellar mass and the extinction. GRB host galaxies tend to be faint $(R>23$; Djorgovski et al. $2001 \mathrm{~b}$ ), and thus, spectroscopic studies of the SED require extensive use of large telescopes. An alternative that allows us to extract information on the properties of the host galaxies is based on multicolour broad band imaging. The main inconvenience of fitting templates to photometric points comes from the insensitivity of the SED solution to the assumed metallicity (Christensen et al. 2004b), which is considered as a secondary parameter (Bolzonella et al. 2000). In practice, in order to determine reliable metallicities for the GRB hosts, spectroscopic observations are required.

The optical afterglow (OA) coordinates $\left(\alpha_{J 2000}=\right.$ $10^{\mathrm{h}} 44^{\mathrm{m}} 59.95, \delta_{J 2000}=+21^{\circ} 31^{\prime} 17^{\prime \prime} .8$ with an uncertainty of 0.5 arcsec in each axis; Price et al. 2003) allowed an extensive follow up from the Northern hemisphere, although it was also visible from the main observatories of the Southern hemisphere. The redshift of GRB 030329 was determined to be $z=0.168$ (Greiner et al. 2003) from high-resolution spectroscopy with the Very Large Telescope (VLT). This makes GRB 030329 the third nearest burst overall (GRB 980425 is the nearest at $z=0.0085$, Galama et al. 1998; and GRB 031203 had $z=0.1055$, Prochaska et al. 2004). As the OA faded, prominent spectral features revealed a Type Ic supernova (named SN 2003dh) peaking 10-15 days after the GRB event (Hjorth et al. 2003; Stanek et al. 2003).

In this paper we present a comprehensive multicolour study of the GRB 030329 host galaxy, similar to those performed for the GRB 000210 (Gorosabel et al. 2003a), GRB 000418
(Gorosabel et al. 2003b) and GRB 990712 (Christensen et al. 2004b) host galaxies. The aim of the analysis is to determine the properties of the stellar populations dominating the optical/near-infrared (NIR) light from the host galaxy and the amount of extinction due to dust in the interstellar medium (ISM) of the host.

Given the faintness of GRB host galaxies in general, detailed observations of the low redshift hosts, as in the present study, may be used to construct a library of spectral templates, applicable (under several assumptions) to the hosts placed at higher redshifts. Furthermore, the case of GRB 030329 is particularly remarkable due to its association with SN 2003dh. Both arguments justify a detailed analysis of this host galaxy.

Throughout this paper, we assume a cosmology where $\Omega_{\Lambda}=0.7, \Omega_{\mathrm{M}}=0.3$ and $H_{0}=65 \mathrm{~km} \mathrm{~s}^{-1} \mathrm{Mpc}^{-1}$. Under these assumptions, the luminosity distance of GRB 030329 is $d_{1}=872.5 \mathrm{Mpc}$ and the look-back time is $2.25 \mathrm{Gyr}$ (15.5\% of the present Universe age).

Section 2 describes the photometric and spectroscopic observations carried out for the GRB 030329 host galaxy. Our main results are reported in Sect. 3. In Sect. 4 we discuss the implications of our work and Sect. 5 summarises the final conclusions.

\section{Observations and analysis}

\subsection{Imaging}

We have used a number of optical/NIR facilities to compile a well sampled SED (see Table 1). The $U B V R$-bands were observed with the 2.56-m Nordic Optical Telescope (NOT) equipped with MOSCA, a $2 \times 2$ mosaic $2048 \times 2048$ pixel CCD, covering a field of view (FOV) of 7!7 $\times 7$ !.7. These observations were carried out in a $2 \times 2$ binning mode, yielding a pixel scale of $0.22 /$ pix.

Additional optical observations were obtained with the BUSCA camera (Reif et al. 2000) at the 2.2-m telescope located at $\mathrm{CAHA}^{1}$. BUSCA allows simultaneous imaging in four broad optical bands. The four channels (hereafter named $C 1$, $C 2, C 3$ and $C 4$ ) are not standard filters and have to be calibrated by observing spectro-photometric standard stars (characteristics of the four BUSCA channels are described in Table 2). $C 1$ resembles the Johnson $U$-band, $C 2$ is a transition between Johnson's $B$ and $V$ filters. $C 3$ is very close to the $R_{\mathrm{c}}$-band and $C 4$ is identical to the $I_{\mathrm{c}}$ filter. Figure 1 shows a coloured composite image based on three out of four BUSCA channels, namely $C 1, C 2$, and $C 4$. BUSCA consists of four $4 \mathrm{~K} \times 4 \mathrm{~K}$ pixel CCDs plus 3 dichroics. The observations were performed in the $2 \times 2$ binning mode. The FOV covered is $12^{\prime} .0 \times 12^{\prime} .0$, and the resulting pixel scale is $0.35 /$ pix.

The $J H K^{\prime}$ observations were carried out with the $3.5-\mathrm{m}$ CAHA telescope equipped with Omega-Prime, a $1024 \times$ 1024 pixel $\mathrm{HgCdTe} \mathrm{CCD}$, which provides a FOV of $6.8 \times 6$ f $^{\prime} 8$ and a pixel scale of 0. '39/pix.

\footnotetext{
${ }^{1}$ Centro Astronómico Hispano Alemán.
} 
Table 1. Optical and NIR photometric observations carried out for the GRB 030329 host galaxy.

\begin{tabular}{lccccc}
\hline \hline $\begin{array}{l}\text { Telescope } \\
(+ \text { instrument })\end{array}$ & Filter & Date UT & $\begin{array}{c}t_{\exp } \\
(\mathrm{s})\end{array}$ & $\begin{array}{c}\text { Seeing } \\
(\operatorname{arcsec})\end{array}$ & $\begin{array}{c}\text { Limiting magnitude } \\
(3 \sigma)\end{array}$ \\
\hline NOT (+MOSCA) & $U$ & $24.03-24.08 / 03 / 04$ & $5 \times 900$ & 1.0 & 25.4 \\
NOT (+MOSCA) & $B$ & $24.85-24.89 / 03 / 04$ & $3 \times 600$ & 1.1 & 25.8 \\
NOT (+MOSCA) & $V$ & $24.86-24.87 / 03 / 04$ & $1 \times 600$ & 1.0 & 24.4 \\
NOT (+MOSCA) & $R$ & $24.87-24.88 / 03 / 04$ & $1 \times 600$ & 0.9 & 24.2 \\
2.2-m (+BUSCA) & $C 1$ & $20.94-21.01 / 03 / 04$ & $19 \times 600$ & $1.8^{\dagger}$ & $25.6^{\star}$ \\
& & $21.97-22.12 / 03 / 04$ & $14 \times 600$ & & \\
& & $22.95-23.06 / 03 / 04$ & $12 \times 600$ & & \\
2.2-m (+BUSCA) & $C 2$ & $20.94-21.01 / 03 / 04$ & $19 \times 600$ & $1.7^{\dagger}$ & $25.1^{\star}$ \\
& & $21.97-22.12 / 03 / 04$ & $14 \times 600$ & & \\
2.2-m (+BUSCA) & $C 3$ & $22.95-23.06 / 03 / 04$ & $12 \times 600$ & & \\
& & $21.97-21.01 / 03 / 04$ & $19 \times 600$ & $1.7^{\dagger}$ & $25.2^{\star}$ \\
2.2-m (+BUSCA) & $C 4$ & $22.95-23.06 / 03 / 04$ & $14 \times 600$ & & \\
& & $20.94-21.01 / 03 / 04$ & $12 \times 600$ & & \\
& & $22.97-22.12 / 03 / 04$ & $14 \times 600$ & $1.6^{\dagger}$ & $24.1^{\star}$ \\
3.5-m (+Omega-Prime) & $J$ & $05.07-05.19 / 01 / 04$ & $113 \times 60$ & 0.7 & \\
3.5-m (+Omega-Prime) & $H$ & $06.07-06.18 / 01 / 04$ & $109 \times 60$ & 0.8 & 24.1 \\
3.5-m (+Omega-Prime) & $K^{\prime}$ & $07.06-07.20 / 01 / 04$ & $99 \times 60$ & 0.8 & 19.7 \\
\hline
\end{tabular}

$\dagger$ Mean seeing of the co-added image.

$\star$ Limiting magnitude obtained co-adding the three epochs.

Table 2. Optical/NIR magnitudes of the host. The fourth and fifth columns show the measured magnitudes in the Vega and AB systems (not corrected for Galactic reddening).

$\dagger 3 \sigma$ upper limit.

\begin{tabular}{lcccc}
\hline \hline $\begin{array}{l}\text { Filter name } \\
\text { (instrument) }\end{array}$ & $\begin{array}{c}\text { Effective } \\
\text { wavelength }(\AA)\end{array}$ & $\begin{array}{c}\text { Bandpass } \\
\text { width }(\AA)\end{array}$ & $\begin{array}{c}\text { Magnitude } \\
(\text { Vega })\end{array}$ & $\begin{array}{c}\text { Magnitude } \\
(\mathrm{AB})\end{array}$ \\
\hline$U$ (MOSCA) & 3638.3 & 396.3 & $22.72 \pm 0.10$ & $23.45 \pm 0.10$ \\
$C 1$ (BUSCA) & 3654.5 & 1081.6 & $23.03 \pm 0.03$ & $23.51 \pm 0.03$ \\
$B$ (MOSCA) & 4358.7 & 680.5 & $23.33 \pm 0.07$ & $23.26 \pm 0.07$ \\
$C 2$ (BUSCA) & 5007.4 & 706.2 & $22.92 \pm 0.05$ & $22.87 \pm 0.05$ \\
$V$ (MOSCA) & 5413.4 & 636.4 & $22.83 \pm 0.10$ & $22.84 \pm 0.10$ \\
$R$ (MOSCA) & 6422.1 & 1072.5 & $22.66 \pm 0.04$ & $22.86 \pm 0.04$ \\
$C 3$ (BUSCA) & 6455.8 & 1147.0 & $22.61 \pm 0.04$ & $22.81 \pm 0.04$ \\
$C 4$ (BUSCA) & 7999.7 & 780.7 & $22.10 \pm 0.04$ & $22.55 \pm 0.04$ \\
$J$ (Omega-Prime) & 12861.5 & 1713.9 & $21.45 \pm 0.16$ & $22.42 \pm 0.16$ \\
$H$ (Omega-Prime) & 16491.6 & 1814.2 & $21.15 \pm 0.24$ & $22.55 \pm 0.24$ \\
$K^{\prime}$ (Omega-Prime) & 21634.9 & 1636.5 & $>19.70 \dagger$ & $>21.57 \dagger$ \\
\hline
\end{tabular}

Reduction was performed following standard procedures running under IRAF ${ }^{2}$. In Table 1 we provide the observing log for our optical and NIR imaging observations.

The optical/NIR imaging was carried out at least 282 days after the GRB, when the $\mathrm{OA}+\mathrm{SN}$ contamination was negligible. The host galaxy appears as a star-like object in all our images, showing NIR and optical profiles consistent with those of field stars. This is consistent with the HST observations by Fruchter et al. (2003), who reported that the host is a dwarf galaxy with a brightness of $V=22.7 \pm 0.3$ (consistent with our $V$-band magnitude, see Table 2). According to the

${ }^{2}$ IRAF is distributed by the National Optical Astronomy Observatories, which are operated by the Association of Universities for Research in Astronomy, Inc., under cooperative agreement with the National Science Foundation. angular scales of the OA-host system discussed in Fruchter et al. (2003), a host compact appearance is expected for seeing conditions above $\sim 0$ '. 5 , in agreement with our observations (seeing $\geq 0$.'7; see Table 1). Therefore, considering that the relative photometry is independent of the aperture radius, the host magnitudes given in Table 2 are based on circular aperture measurements (PHOT running under IRAF) with no aperture corrections and an aperture radius of 1.5 times the full width at half maximum (FWHM).

The $U B V R$-band calibration is based on Henden (2003) and the $J H K^{\prime}$ calibration on Guziy et al. (2005). For the BUSCA data, the flux calibration was carried out by observing the spectro-photometric standard star GD153 (Bohlin et al. 1995) at the same airmass to that of the GRB host on a photometric night. This allowed us to establish secondary field standard 


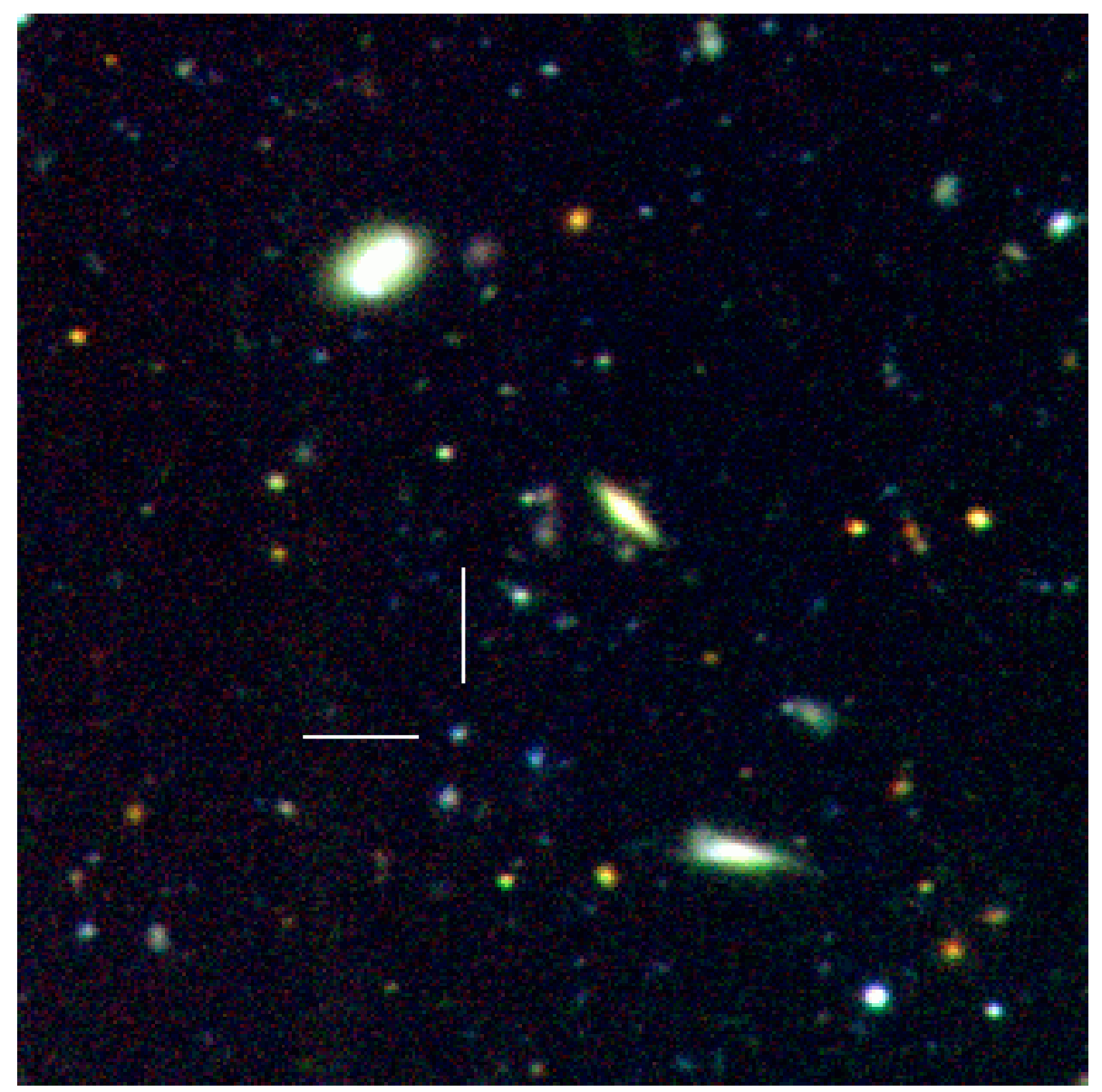

Fig. 1. The field of the GRB 030329 host galaxy. The picture is a coloured composite image created by combining three BUSCA images taken in the $C 1, C 3$ and $C 4$ bands. The FOV is $2^{\prime} .0 \times 2^{\prime} \cdot 0$. The location of the host galaxy is indicated. As can be seen from the picture, the host shows a bluish colour. North is upwards and East is leftwards.

stars in the AB system. As can be seen in Fig. 3, the agreement of the BUSCA calibration with the NOT optical magnitudes is satisfactory.

\subsection{Spectroscopy}

Spectroscopy of the host of GRB 030329 was obtained on 2003 June 19 with the Focal Reducer and Low Dispersion Spectrograph (FORS2) instrument ${ }^{3}$ attached to the 8.2-m UT4 of the European Southern Observatory Very Large Telescope. The FORS2 instrument is equipped with two $2048 \times 4096$ pixel MIT CCDs with $15 \mu \mathrm{m}$ pixels. We used the standard resolution mode that gives a FOV of 6 ' $^{\prime} \times 6$ ' .8 , and with $2 \times 2$ binning on the chip we obtained a pixel size on the sky of 0.25 .

The observations were conducted with the $300 \mathrm{~V}$ grism and an order sorting filter GG375, which efficiently covers the wavelength range from $\sim 3800-8800 \AA$. A 1". 3 wide slit was used and the seeing during the observations was typically below $0 .{ }^{\prime} 6$, yielding a spectral resolution of $\sim 10.5 \AA$. Given the small size of the object, the entire flux of the host galaxy should thus be included in the slit. A position angle of 123.6 degrees was used to align a nearby star on the slit (see Hjorth et al. 2003). Three spectra of $900 \mathrm{~s}$ each were obtained,

\footnotetext{
${ }^{3}$ http://www.eso.org/instruments/fors/
}

with small offsets on the slit between the individual exposures. The airmass was always quite substantial since these observations were obtained from the Southern hemisphere, and varied from 1.8 to 2.3 . The final combined spectrum is shown in Fig. 2.

The spectra were reduced in a standard way, including bias subtraction, flat fielding, and wavelength calibration using spectra of a Helium-Argon lamp. Flux calibration was done relative to the spectrophotometric standard star Feige67 (Oke 1990). The absolute flux calibration of the combined spectrum was obtained relative to our broad band photometry.

We note that our host galaxy spectroscopic observations were carried out $\sim 82$ days after the GRB, when the contribution of the $\mathrm{OA}+\mathrm{SN}$ and the host were comparable (Guziy et al. 2005). In contrast, the host properties derived by Matheson et al. (2003) are based on spectra acquired only 6-10 days after the GRB, when the OA+SN was still 50-25 times brighter than the host. This intense overimposed radiation field might affect the measurement of the host emission lines fluxes (especially the faint ones), so we consider our spectrum a cleaner probe of the host galaxy physical properties.

Before using the emission lines as indicators of several host galaxy properties, they were corrected for Galactic foreground reddening in the direction of the GRB $(E(B-V)=0.025$; 


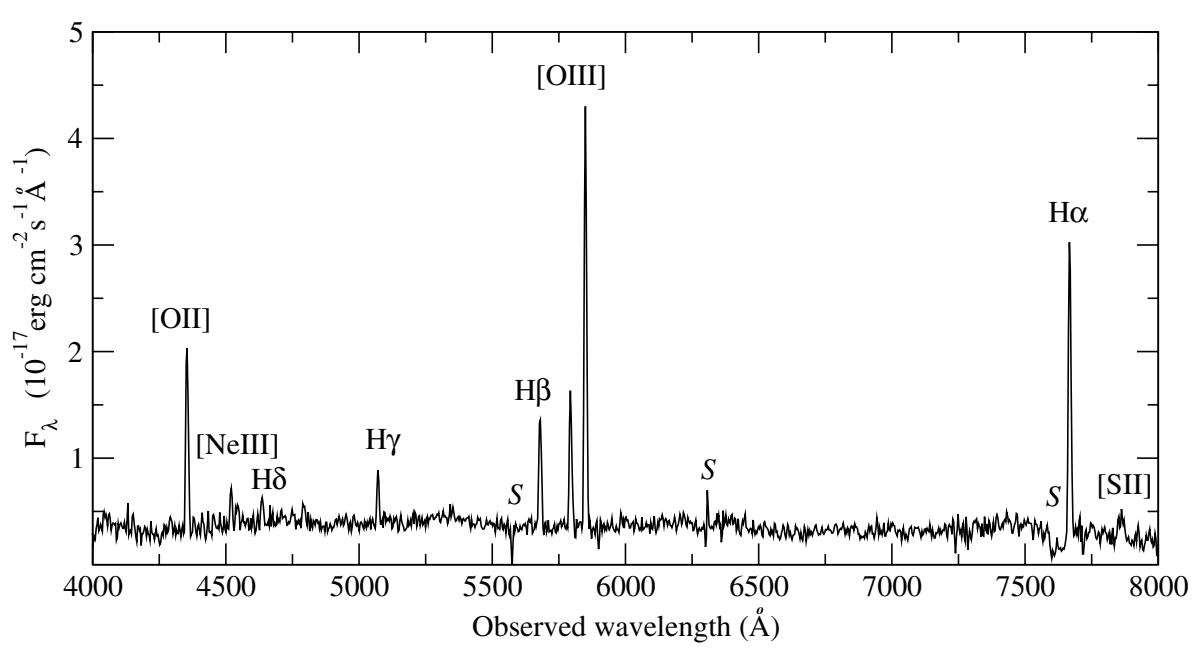

Fig. 2. The GRB 030329 host galaxy VLT spectrum. The flux is corrected for Galactic reddening $(E(B-V)=0.025)$ considering a MW extinction law (Cardelli et al. 1989). The residuals resulting from the telluric line subtractions are indicated by an $S$. Note the telluric line affecting the left wing of $\mathrm{H} \alpha$. The spectrum was acquired $\sim 82$ days after the GRB, when the contribution to the continuum by the OA+SN was still significant.

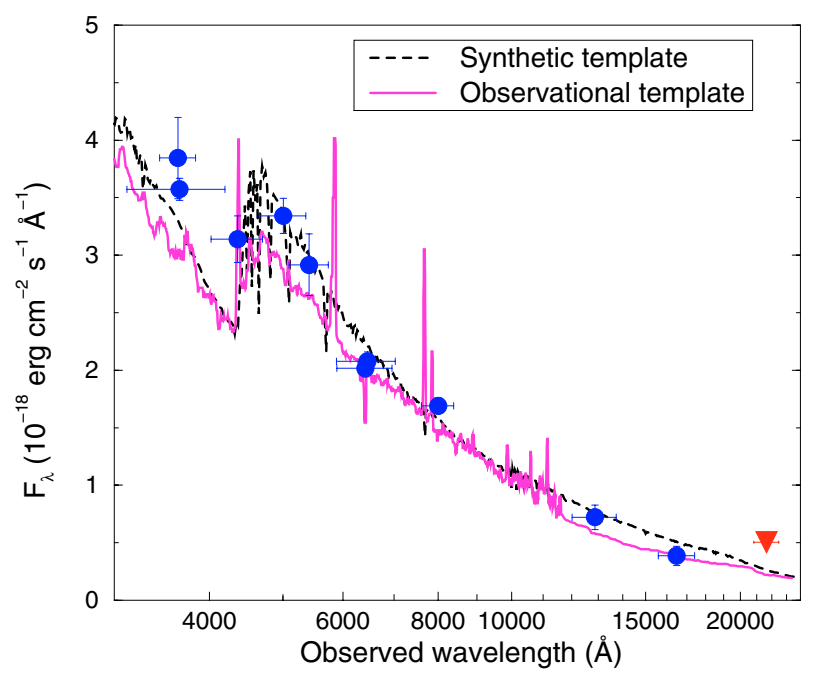

Fig. 3. The optical/NIR SED of the GRB 030329 host galaxy. The circles show the $U B V R, C 1, C 2, C 3, C 4$ and $J H$-band detections. The filled triangle represents the $K^{\prime}$ upper limit. The abscissa is represented on a logarithmic scale in order to enlarge the region around the $4000 \AA$ break. The dashed line shows the fit obtained assuming a metallicity of $Z=0.004$, a S55 type IMF and a Prevot et al. (1984) extinction law. Note the similarity with the Stb1 K96 empirical template overplotted with a continuous line.

Schlegel et al. 1998) considering a MW extinction law (Cardelli et al. 1989).

\subsection{Photometry: constructing the optical/NIR SED}

In order to derive the corresponding effective wavelengths and $\mathrm{AB}$ magnitudes we convolved each filter transmission curve plus the corresponding CCD efficiency curve (see Table 2 ) with the Vega spectrum ( $\alpha$ Lyrae, $m=0$ in all bands by definition; Bruzual \& Charlot 1993). The fit of the SED has been mainly obtained using synthetic templates, but checks have also been performed using observational templates (Kinney et al. 1996, hereafter K96).

The synthetic SED analysis is based on the code Hyper ${ }^{4}$ (Bolzonella et al. 2000). Eight synthetic spectral types were used representing Starburst galaxies (Stb), Ellipticals (E), Lenticulars (S0), Spirals (Sa, Sb, Sc and Sd) and Irregular galaxies (Im). Each spectral type has associated a $\operatorname{SFR}(t)$ temporal history, and hence a characteristic time scale $(S F R \propto$ $\exp (-t / \tau)$, where $\tau$ can range from 0 for Stb to $\infty$ for Im galaxies). For the generation of the synthetic templates, two initial mass functions (IMFs) have been considered: Salpeter (1955, S55) and Chabrier (2003, CH03). Four extinction laws have been taken into account: Calzetti et al. (2000, suitable for Stbs), Seaton (1979, for the Milky Way, MW), Fitzpatrick (1986, for the Large Magellanic Cloud, LMC) and Prévot et al. (1984, for the Small Magellanic Cloud, SMC). The construction of the HyperZ templates has been performed using the GALAXEV public code (Bruzual \& Charlot 2003), using as input the metallicity ( $Z$, see Sect. 3.4) derived from our VLT spectroscopic observations.

In addition to the aforementioned evolutionary templates, the observational templates from K96 were considered. These extra spectral templates give complementary information on the galaxy type and extinction. The observed K96 templates can be grouped into seven sets: Bulge (B), E, S0, Sa, Sb, Sc and Stb galaxies. Additionally, the Stb K96 templates are subdivided into six classes depending on their extinction by increasing $E(B-V)$ in steps of 0.1 (from Stb1 to Stb6; see more details in K96). The extinction for the B, E, S0, Sa, Sb and Sc templates is a free parameter.

For the construction of the photometric SED all the magnitudes were corrected for Galactic foreground reddening along the GRB line of sight (Schlegel et al. 1998) assuming a typical MW extinction law (Cardelli et al. 1989).

\footnotetext{
${ }^{4}$ http://webast.ast.obs-mip.fr/hyperz/
} 
Table 3. Emission lines detected in the GRB 030329 host galaxy VLT spectrum. The fluxes are corrected for Galactic extinction.

\begin{tabular}{lccc}
\hline \hline Element & $\begin{array}{c}\text { Flux } \\
\left(10^{-17} \mathrm{erg} \mathrm{cm}^{-2} \mathrm{~s}^{-1}\right)\end{array}$ & $\begin{array}{c}\text { Observed } \\
\text { wavelength }(\AA)\end{array}$ & Redshift \\
\hline$[\mathrm{O}$ II $]$ & $20.40 \pm 0.39$ & 4354.08 & 0.1681 \\
{$[\mathrm{Ne}$ III $]$} & $4.04 \pm 0.28$ & 4520.15 & 0.1682 \\
$\mathrm{H} \delta$ & $2.33 \pm 0.20$ & 4792.96 & 0.1684 \\
$\mathrm{H} \gamma$ & $4.82 \pm 0.20$ & 5071.08 & 0.1683 \\
$\mathrm{H} \beta$ & $11.70 \pm 0.36$ & 5679.70 & 0.1683 \\
{$[\mathrm{O}$ III $]$} & $14.76 \pm 0.38$ & 5793.80 & 0.1684 \\
{$[\mathrm{O}$ III $]$} & $42.21 \pm 0.31$ & 5849.79 & 0.1684 \\
$\mathrm{H} \alpha$ & $32.40 \pm 0.47$ & 7667.55 & 0.1683 \\
{$[\mathrm{~S} \mathrm{II}]$} & $3.27 \pm 0.32$ & 7861.65 & 0.1684 \\
\hline
\end{tabular}

\section{Results}

The VLT spectrum allows us to estimate the host galaxy extinction $\left(A_{\mathrm{v}}\right)$, the metallicity $(Z)$ and the SFR, based on the oxygen and Balmer emission lines. Table 3 displays the list of emission lines detected in our VLT spectrum. The flux of the lines, with the exception of $\mathrm{H} \delta$, are consistent within $1.5 \sigma$ with the fluxes previously reported for the GRB 030329 host galaxy (Hjorth et al. 2003).

Alternatively, the SED constructed from the optical/NIR photometric points gives an independent estimate of the extinction and the SFR. The SED also provides us with information on the stellar population age, the stellar mass and on the host galaxy absolute luminosity. For self-consistency, we based our SED fitting analysis on templates constructed adopting the metallicity derived from our spectroscopic study $(Z=0.004)$. Table 2 shows the optical/NIR magnitudes given in the Vega and $\mathrm{AB}$ systems, not corrected for Galactic or host reddening. The listed magnitude errors account for both the zero point and the statistical errors. Figure 3 shows our photometric points and the SED solution obtained for a SMC extinction law, a S55 IMF and a metallicity of $Z=0.004$. Considering all the possible extinction laws (4) and IMFs (2), we end up with 8 possible templates for each galaxy type.

\subsection{Photometric redshift}

Given that the spectroscopic redshift is known, we only use the photometric redshift to check our fitted SEDs for internal consistency. The spectroscopic redshift falls within the $68 \%$ uncertainty region of the photometric redshift in 6 out of the $8 \mathrm{IMF} /$ extinction law combinations.

A rough estimate based on the K96 templates yields a photometric redshift around $z \sim 0.11$, reasonably close to the spectroscopic redshift. We consider the inferred photometric redshifts to be consistent with the spectroscopic redshift and hereafter we fix the redshift of the SEDs to $z=0.168$.

\subsection{Absolute magnitude}

Subluminous galaxies are defined as having luminosity values below the knee of the luminosity function given by $L^{\star}$ (or the equivalent $M_{B}^{\star}$ ). Slightly different values for $M_{B}^{\star}$ have been
Table 4. Main results obtained based on the empirical templates by Kinney et al. (1996). The templates have been ordered according to their colour, bluer on the top (Stb1) and redder at the bottom (B). There is a clear correlation between the goodness of the fit and the colour of the template: the bluer the colour the lower $\chi^{2} /$ d.o.f.

\begin{tabular}{lcc}
\hline \hline $\begin{array}{l}\text { Galaxy } \\
\text { Template }\end{array}$ & $\begin{array}{c}\chi^{2} / \text { d.o.f. } \\
\text { (d.o.f. }=9)\end{array}$ & $E(B-V)$ \\
\hline Stb1 & 3.06 & $0.05 \pm 0.05^{\dagger}$ \\
Stb2 & 3.01 & $0.15 \pm 0.05^{\dagger}$ \\
Stb3 & 14.86 & $0.30 \pm 0.05^{\dagger}$ \\
Stb4 & 24.48 & $0.45 \pm 0.05^{\dagger}$ \\
Stb5 & 36.77 & $0.55 \pm 0.05^{\dagger}$ \\
Stb6 & 47.57 & $0.65 \pm 0.05^{\dagger}$ \\
Sc & 3.47 & 0.18 \\
Sb & 133.38 & 0 \\
Sa & 153.72 & 0 \\
S0 & 176.87 & 0 \\
E & 182.18 & 0 \\
B & 180.96 & 0 \\
\hline
\end{tabular}

$\dagger$ Extinction fixed by the template definition.

reported depending on the galaxy colour and type (e.g., Schechter 1976; Lilly et al. 1995). For simplicity and in order to perform a comparative study with the C04A results, we have assumed the same value of $M_{B}^{\star}=-21$ as adopted by C04A.

According to the synthetic templates, we estimate a mean $B$-band absolute magnitude of $M_{B}=-16.5$, similar to that of the SMC. This value is largely independent of the assumed extinction law, IMF and metallicity. The $M_{B}=-16.5$ value corresponds to a luminosity of $L \sim 0.016 L^{\star}$. We conclude that the GRB 030329 host is clearly a subluminous galaxy. This is consistent with the host luminosity inferred by Fruchter et al. (2003).

\subsection{Estimating $A_{v}$}

Among the 8 possible extinction law and IMF combinations, we find for $50 \%$ of the cases an extinction value within the $0.6<A_{\mathrm{v}} \leq 0.7$ bin. For these four combinations no clear preferential extinction law or IMF is found. The rest of the inferred $A_{\mathrm{v}}$ values are equally distributed in the $0.7<A_{\mathrm{v}} \leq 0.8$ and $A_{\mathrm{v}} \leq 0.6$ regions. The mean extinction value derived for the synthetic templates is $A_{\mathrm{v}}=0.6$.

We have also used the empirical templates by K96 to verify the extinction value obtained with the synthetic templates. The best fit is then obtained with the blue Stb1 and Stb2 SEDs (see Table $4, \chi^{2} /$ d.o.f. $\sim 3$ ), which are defined to have the lowest extinctions among the K96 Stb sample $(0<E(B-V)<0.21)$. Stb templates with larger $E(B-V)$ values clearly yield worse results. To translate the value of $E(B-V)$ to $A_{\mathrm{v}}$, we have adopted a value of $A_{\mathrm{v}} / E(B-V)=3.1$. Thus, the Stb1 and Stb2 templates imply a value of $0<A_{\mathrm{v}}<0.65$, in agreement with the mean $A_{\mathrm{v}}=0.6$ inferred based on the synthetic templates. The assumed $A_{\mathrm{v}} / E(B-V)$ fraction is indicative (the actual $A_{\mathrm{v}} / E(B-V)$ value depends on the unknown host extinction law) and is used to check the $A_{\mathrm{v}}$ value inferred with the synthetic templates. 
Figure 3 shows the similarities of the Stb1 empirical template and the solution obtained by an evolutionary SED. Leaving $E(B-V)$ as a free parameter for the non Stb templates, we obtained the $E(B-V)$ values reported in Col. 3 of Table 4 . These fits are not satisfactory. Higher values of $E(B-V)$ for these templates would make the spectrum even redder and hence provide even larger $\chi^{2} /$ d.o.f. values. The exception is the Sc template which presents a value of $\chi^{2} /$ d.o.f. $=3.47$, just slightly larger than for the Stb1 and Stb2 templates. The $E(B-V)$ inferred for the Sc template is actually consistent with the one derived for the empirical Stb1 and Stb2 templates, as well as with that derived for the synthetic SEDs.

In principle, the relative fluxes of the Balmer lines can be used as an indicator of the intrinsic host galaxy $A_{\mathrm{v}}$ (Osterbrock 1989). The $\mathrm{H} \alpha$ emission line is affected by a nearby telluric band, so we have used the rest of the Balmer lines present in our spectrum $(\mathrm{H} \beta, \mathrm{H} \gamma, \mathrm{H} \delta)$ to estimate $A_{\mathrm{v}}$. A major difficulty in using the faint Balmer lines comes from the potential contribution of an underlying evolved stellar population. This can introduce absorption features with equivalent widths ( $E W \mathrm{~s})$ up to a few $\AA$ (Bruzual \& Charlot 2003), which are comparable to our measured emission fluxes in $\mathrm{H} \gamma$ and $\mathrm{H} \delta$. We have averaged the $\mathrm{H} \gamma$ and $\mathrm{H} \delta$ absorption $E W$ s of the 8 galaxy templates fitted to our photometric points and corrected the emission lines fluxes to estimate $A_{\mathrm{v}}$. The mean $\mathrm{H} \gamma$ and $\mathrm{H} \delta$ restframe absorption $E W$ s were $8 \pm 2 \AA$ and $7 \pm 3 \AA$, respectively. The extinction values inferred are $A_{\mathrm{v}}=0.33 \pm 0.19$ and $A_{\mathrm{v}}=0.86 \pm 0.22$, based on $\mathrm{H} \gamma$ and $\mathrm{H} \delta$, respectively. The weighted mean of the two extinction values is $A_{\mathrm{v}}=0.56 \pm 0.14$, consistent with the $A_{\mathrm{v}}=0.6$ value previously inferred based on the synthetic and empirical galaxy templates. We note that the $A_{\mathrm{v}}$ values determined through $\mathrm{H} \gamma$, and especially from $\mathrm{H} \delta$, are sensitive to the correction for the underlying population. Therefore, although the agreement is very good, we will assume the extinction determined with the synthetic SED templates and use $A_{\mathrm{v}}=0.6$ throughout the paper.

\subsection{Estimate of the metallicity}

The detections of [O II], [O III] and $\mathrm{H} \beta$ in emission allow us to estimate the host metallicity based on the $R_{23}$ iterative technique (e.g., Kewley \& Dopita 2002). Correcting the emission line fluxes for $A_{\mathrm{v}}=0.6$ and assuming a SMC extinction law, we obtain a metallicity of $Z=0.004\left(Z=0.20 Z_{\odot}\right.$, where $\left.Z_{\odot}=0.02\right)$ and an ionization parameter $q=6.84 \times 10^{7} \mathrm{~cm} \mathrm{~s}^{-1}$. The inferred metallicity is well below $Z_{\odot}$, implying that the GRB 030329 host is a low metallicity galaxy.

The inferred metallicity is largely insensitive to the assumed extinction law. Typical MW and LMC extinction laws (Pei 1992) yield metallicity values only $\sim 3 \%$ away from the metallicity derived based on the SMC.

Hjorth et al. (2003) reported a rough metallicity value of $[\mathrm{O} / \mathrm{H}]=-1.0$ (equivalent to $Z=0.10 Z_{\odot}$ ), not correcting for Galactic reddening, intrinsic host galaxy reddening and underlying population. If the abovementioned corrections are not applied to our measurements, we obtain a metallicity value of $[\mathrm{O} / \mathrm{H}]=-0.8\left(Z=0.16 Z_{\odot}\right)$. Given that the typical metallicity uncertainty of the $R_{23}$ technique is \pm 0.2 dex (Kewley \& Dopita 2002), we conclude that our metallicity estimate is in agreement with the value inferred by Hjorth et al. (2003).

The $R_{23}$ diagnostic has two values (lower and upper branch), providing two possible metallicity values. In this study we assume the metallicity inferred based on the $R_{23}$ lower branch. According to Sollerman et al. (2005) the $R_{23}$ upper branch yields a metallicity value of $[\mathrm{O} / \mathrm{H}]=-0.1$. This metallicity degeneracy can be broken by considering other emission lines such as [N II] which was not detected in our VLT spectrum. As noted by Sollerman et al. (2005), the [N II] flux upper limit derived from the VLT spectrum is not constraining enough to resolve the $R_{23}$ degeneracy.

However, the $R_{23}$ upper branch predicts a rather bright galaxy $\left(M_{B} \sim-19\right.$; Lee et al. 2003), while the lower branch metallicity is more consistent with the $M_{B}$ magnitude of the host $\left(M_{B} \sim-16\right.$ is expected for $\left.Z=0.20 Z_{\odot}\right)$, as pointed out by Sollerman et al. (2005). Therefore, the lower branch solution is more likely than the upper one, but a host metallicity close to solar abundance cannot be completely excluded.

\subsection{Estimate of the SFR}

Following the standard $\mathrm{H} \alpha$ and [O II] diagnostic techniques (Kennicutt 1998, hereafter K98) the SFR can be estimated. However, given the contamination that $\mathrm{H} \alpha$ suffers from telluric lines, we have used the SFR given by the [O II] diagnostic. This gives a $S F R=0.26 M_{\odot} \mathrm{yr}^{-1}$ (corrected for Galactic extinction but not for host galaxy extinction), in agreement with the SFR derived by Hjorth et al. (2003, SFR $=0.20 \pm 0.07 M_{\odot} \mathrm{yr}^{-1}$ once it is corrected for Galactic extinction). For a Pei (1992) SMC-like extinction law, and adopting $A_{\mathrm{v}}=0.6$, we obtain an unextinguished SFR of $0.6 M_{\odot} \mathrm{yr}^{-1}$.

A verification of the SFR inferred from the [O II] line can be carried out estimating three independent SFRs through $\mathrm{H} \beta$, $\mathrm{H} \delta$ and $\mathrm{H} \gamma$ assuming a typical case $\mathrm{B}$ recombination and taking into account the extinction previously derived. The inferred three SFRs show a mean weighted value of $0.63 M_{\odot} \mathrm{yr}^{-1}$ and a dispersion of $0.15 M_{\odot} \mathrm{yr}^{-1}$. This is fully consistent with the SFR derived based on [O II].

The photometric SEDs are an additional diagnostic to estimate the SFR. The method to derive the SFR from the UV continuum flux is one of several diagnostic methods used in the literature to measure SFRs in galaxies (see K98 for a comprehensive review). Obviously, if there is dust-enshrouded star formation, this UV-based method will only provide a lower limit to the actual SFR. At $z=0.168$ the $2800 \AA$ region is redshifted to $\sim 3270 \AA$, so it is included in the sensitivity region of the $U$-band and the BUSCA $C 1$ band. We have derived the average value of the fluxes in both filters $\left(F_{2800 \AA} \sim 1.5 \times 10^{-29} \mathrm{erg} \mathrm{s}^{-1} \mathrm{~Hz}^{-1} \mathrm{~cm}^{-2}\right)$ and determined the UV luminosity at restframe $2800 \AA$ to be $L_{\mathrm{UV}} \sim 1.2 \times$ $10^{27} \mathrm{erg} \mathrm{s}^{-1} \mathrm{~Hz}^{-1}$. Following K98 this corresponds to a $S F R=$ $0.17 M_{\odot} \mathrm{yr}^{-1}$. Given that the rest frame luminosity of the host is $L=0.016 L^{\star}$, we obtain a specific star formation (SSFR) rate of $S S F R=10.6 M_{\odot} \mathrm{yr}^{-1}\left(L / L^{\star}\right)^{-1}$. If we correct 
the previous UV SFR and SSFR estimates for the reddening effect in the host galaxy $\left(A_{\mathrm{v}} \sim 0.6\right.$, assuming a SMC extinction law), we obtain an unextinguished $S F R \sim 0.54 M_{\odot} \mathrm{yr}^{-1}$ and $S S F R \sim 34 M_{\odot} \mathrm{yr}^{-1}\left(L / L^{\star}\right)^{-1}$. Thus, the unextinguished SFR derived from the UV continuum is in agreement with the unextinguished SFR inferred based on the [O II] and the Balmer lines $\left(S F R \sim 0.6 M_{\odot} \mathrm{yr}^{-1}\right)$. Given that C04A used the UV diagnostic technique for their SSFR estimates, for comparison we adopted the SSFR derived from the UV continuum $\left(\sim 34 M_{\odot} \mathrm{yr}^{-1}\left(L / L^{\star}\right)^{-1}\right)$.

\subsection{A potential galaxy association at $z \sim 0.17$ ?}

A GRB 030329 field galaxy located at $\alpha_{J 2000}=10^{\mathrm{h}} 45^{\mathrm{m}} 01.46^{\mathrm{s}}$,

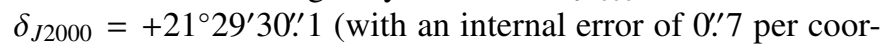
dinate) was accidentally placed on the FORS 2 slit. The galaxy showed prominent emission lines similar to the host galaxy, and turned out to be at a redshift of $z=0.1710 \pm 0.0003$. The similarity to the GRB host galaxy redshift raises the question of a potential clustering or association at $z \sim 0.17$. The nearby galaxy is located 192.4 arcsec from the host, implying a distance of $\sim 600 \mathrm{kpc}$ at the host galaxy redshift. The radii of galaxy clusters is of the order of a few Mpc, so the measured angular distance to the host galaxy is compatible with a physical association at $z \sim 0.17$. On the other hand, the velocity difference is $\sim 685 \pm 104 \mathrm{~km} \mathrm{~s}^{-1}$, in agreement with the velocity dispersions measured in $z \lesssim 0.15$ galaxy clusters (Desai et al. 2004).

A spectroscopic study of a significant galaxy sample in the GRB field would be necessary to investigate whether the host is physically linked to other angularly close galaxies. If such an association is confirmed, the case of the GRB 030329 host would be opposite to the preferential tendency of GRB hosts to reside in low galaxy density environments (Bornancini et al. 2004).

\subsection{Galaxy type}

When using the synthetic templates for the fit, we found that the favoured galaxy type is not very dependent on the assumed IMF and extinction law. Among the $8 \mathrm{IMF} /$ extinction law combinations, a starburst galaxy is obtained in $50 \%$ of the cases. The rest of the cases are distributed in Sa (25\%), S0 (12.5\%) and $\mathrm{Sb}(12.5 \%)$. Thus the synthetic SED fits favour a Stb type.

This result is supported by the fits obtained with the empirical templates by K96 (Table 4). In general the starburst templates give lower values for $\chi^{2} /$ d.o.f. when fitting the SED. The only exception is for the Sc type, $\chi^{2} /$ d.o.f. $=3.47$, whose value is quite close to the best ones obtained for the Stb1 and Stb2 templates.

\subsection{Dominant stellar population age}

The inferred mean stellar population age for the $8 \mathrm{IMF} /$ extinction law combinations is $150 \pm 80 \mathrm{Myr}$. In principle progenitors older than $\sim 50 \mathrm{Myr}$ are not obvious to accommodate within the collapsar scenario, since the age of an $\sim 8 M_{\odot}$ star (the minimum SN progenitor mass) when it explodes as a core-collapse $\mathrm{SN}$ is $\sim 50 \mathrm{Myr}$ (see for instance Portinari et al. 1998).

However, an ideal starburst where all the star formation occurs simultaneously, and hence all the stars have exactly the same age, is not realistic. Thus, in a real starburst like the GRB 030329 host, although the majority of the star formation occurred $\sim 150 \mathrm{Myr}$ ago, the star formation cannot stop sharply, but has a stellar age dispersion which spreads down to $150 \mathrm{Myr}$. Hence, an age of $\sim 150 \mathrm{Myr}$ should be considered the upper limit of the progenitor age.

An independent check of the dominant stellar population age can be performed comparing the restframe $\mathrm{H} \beta$ equivalent width derived from the VLT spectrum $(E W \sim 50 \AA$, corrected for the underlying population) to the one predicted by Starburst99 (SB99; Leitherer et al. 1999). According to SB99, for a metallicity of $Z=0.004$ our $\mathrm{H} \beta E W$ corresponds to ages ranging from $\sim 10 \mathrm{Myr}$ (for the case of an ideal instantaneous $\mathrm{SFR}, \tau=0$ ) to $\sim 300 \mathrm{Myr}$ (for a constant SFR, $\tau=\infty$ ). Our age of $150 \pm 80 \mathrm{Myr}$ is consistent with this age range, supporting that the star formation might be brief, but not perfectly instantaneous $(\tau=0)$.

\subsection{Host galaxy stellar mass}

Given that the Bruzual \& Charlot (2003) templates are normalized to one Solar mass, the normalization factor used to scale the templates to our photometric fluxes allowed us to estimate the host galaxy stellar mass $\left(M_{\text {host }}\right)$. We caution however that the optical photometric points are likely dominated by young massive stars, so this estimator tends to be insensitive to the redder low-luminosity (but very abundant) low-mass underlying stellar population. This effect is especially relevant in galaxies, like the GRB 030329 host, where the optical light is dominated by a bright starburst episode(s). So, strictly speaking, the derived mass should be considered a lower limit to the total stellar mass present in our host.

Assuming the S55 and CH03 IMFs and not accounting for the effect of the internal extinction, we derived mass values of $M_{\text {host }}=9.1 \pm 1.3 \times 10^{7} M_{\odot}$ and $M_{\text {host }}=6.0 \pm 0.9 \times 10^{7} M_{\odot}$, respectively. Given that an extinction value of $A_{\mathrm{v}}=0.6$ implies a scale up of the intrinsic optical flux by a factor of $\sim 2$, we adopt $10^{8} M_{\odot}$ as a conservative lower limit to the total stellar mass.

An independent rough estimation of this value can be obtained through the statistical mass-to-luminosity ratios $(M / L)$ reported for the galaxies of the Sloan Digitized Sky Survey (SDSS; York et al. 2000). Adopting $K=K^{\prime}$, for the $K$-band absolute magnitude $\left(M_{K}=-19.1\right.$, basically not affected by the host galaxy reddening if $A_{\mathrm{v}} \sim 0.6$ is assumed) and the $B-R$ restframe colour of our host, $M / L \sim 0.7$ is expected (see Table 7 of Bell et al. 2003). This would imply a stellar mass of $M_{\text {host }} \sim 7 \times 10^{8} M_{\odot}$. The $M / L$-colour relations derived by Bell et al. (2003) are based on a large sample of galaxies with no distinction of the galaxy type, so they represent an average over a wide morphological range. However, for a given colour, low-mass galaxies tend to show lower 
$M / L$ values (Binney $\&$ Merrifield 1998 ), so $7 \times 10^{8} M_{\odot}$ is a plausible upper limit to the host galaxy stellar mass. Therefore, we conclude that the total stellar mass of our galaxy should be $10^{8} M_{\odot} \lesssim M_{\text {host }} \lesssim 7 \times 10^{8} M_{\odot}$.

\section{Discussion}

The results above were obtained assuming the $R_{23}$ low metallicity branch (see Sollerman et al. 2005). To verify that our results are not critically dependent on this assumption we have also run models with Solar abundances.

No remarkable differences were found in the output parameters. This is in agreement with the extensive tests performed by Bolzonella et al. (2000) who reported that the metallicity is a secondary parameter for the SED fitting technique

The most relevant impact of the metallicity is the effect on the stellar age. Solar metallicity templates show slightly redder colours than the $Z=0.004$ templates, therefore less evolution is required to fit the photometric points, yielding younger stellar ages. Thus, the mean stellar population age inferred using Solar metallicity templates was $\sim 43 \pm 7 \mathrm{Myr}$, a factor of three lower than the one inferred with the $Z=0.004$ templates.

The metallicity showed a second order impact on $A_{\mathrm{v}}$. Given that the Solar metallicity templates are redder, they provide lower $A_{\mathrm{v}}$ values. Thus, assuming Solar metallicity we obtain an extinction of $A_{\mathrm{v}}=0.4$ (instead of $A_{\mathrm{v}}=0.6$ for $Z=0.004$ ), yielding an UV unextinguished $S F R=0.37 M_{\odot} \mathrm{yr}^{-1}$ and SSFR $=23.1 M_{\odot} \mathrm{yr}^{-1}\left(L / L^{\star}\right)^{-1}$. The rest of the parameters (photometric redshift, galaxy type, stellar mass) are insensitive to the metallicity of the synthetic templates.

\subsection{The GRB 030329 host SSFR relative to galaxies at a similar redshift}

We performed a comparative analysis of the GRB 030329 host SSFR with respect to the SSFRs obtained for a galaxy sample based on the SDSS. In order to minimize any impact that the SSFR-redshift evolution (Cowie et al. 1996; Juneau et al. 2005) might have on our analysis, only galaxies with $0.160<z<0.175$ were considered. Following the process explained in C04A, the unextinguished SSFR of the SDSS galaxies were calculated using HyperZ. We then selected only the galaxies that showed acceptable SED fits $\left(\chi^{2} /\right.$ d.o.f. $\left.\leq 1.5\right)$. To estimate the probability errors, the process was repeated for the two IMFs given by $\mathrm{S} 55$ and $\mathrm{CH} 03$.

Assuming an S55 IMF, among the 12333 SSFRs calculated for the SDSS sample, only 753 showed SSFRs larger than $34 M_{\odot} \mathrm{yr}^{-1}\left(L / L^{\star}\right)^{-1}$, implying only $6.1 \%$ of the sample. The results did not change qualitatively when the $\mathrm{CH} 03$ IMF was used $(6.9 \%)$. Hence we estimate that the GRB 030329 host SSFR is higher than $\sim 93.5 \%$ of the galaxies at an equivalent redshift.

This result is also supported by an independent (but more reduced) comparison sample based on 1067+1611 HDF galaxies (adding the catalogues by Fernández-Soto et al. 1999, and Vanzella et al. 2001). For a S55 IMF, only one of the 89 HDF galaxies in the $0.05<z<0.25$ redshift slot showed a SSFR larger than the one of the GRB 030329 host (98.9\% percentile).
If a redshift $0<z<0.25$ window is assumed (containing 143 galaxies), then the GRB 030329 host SSFR is over 97.9\% of the sample. Again the conclusions remain largely invariant for a CH03 IMF (in a $0<z<0.25$ redshift window only 5 among 136 galaxies displayed $S S F R>34 M_{\odot} \mathrm{yr}^{-1}\left(L / L^{\star}\right)^{-1}$; $96.3 \%$ percentile).

\subsection{The GRB 030329 host in the framework of previous compilations}

The SFR (corrected for intrinsic extinction) of the GRB 030329 host is the lowest in the C04A sample. However, the SSFR is the highest, given that the $M_{B}$ of our host is 1.6 mag dimmer than the faintest C04A sample galaxy. The GRB 030329 host falls into the selection criteria defined by C04A $(R<25.3$, known redshift, detection in 5 or more filters, not very complex morphology). This allowed us to incorporate our host into the C04A 10 host galaxy sample and repeat part of the C04A statistical analysis.

In order to construct a reference sample with similar spatial distribution to the $(10+1)$ hosts, we calculated the SSFR only for the HDF galaxies with redshifts consistent with our 11 host galaxies $(0.17 \leq z \leq 2.1)$. Selecting only HDF galaxies with acceptable SED fits $\left(\chi^{2} /\right.$ d.o.f. $\left.\leq 1.5\right)$ reduced the HDF catalogues to 1917 for a S55 IMF, and 1880 for a CH03 IMF.

The SSFR distribution of our 11 hosts and the HDF reference samples were statistically compared using the two-sample Kolmogorov-Smirnov test (Peacock 1983; see C04A for more details). The probability that the two samples share the same parent SSFR distribution ranges from $p=1.2 \times 10^{-4}$ (S55) to $p=5.5 \times 10^{-4}(\mathrm{CH} 03)$ depending on the assumed IMF. This calculation reduces the C04A $p$ values by an order of magnitude and strengthens the previous C04A results, who concluded that GRB hosts show on average higher SSFRs than HDF galaxies at similar redshifts.

According to our synthetic SED fitting a $K^{\prime}$-band magnitude of $K^{\prime} \sim 20.5$ is predicted, implying $R-K \sim 2.1$ (adopting $K=K^{\prime}$ and correcting for Galactic reddening). This colour is consistent with the colours of the host galaxy sample by Le Floc'h et al. (2003). The $K$-band absolute magnitude estimated for the host $\left(M_{K}=-19.1\right)$ is in agreement with the general results by Le Floc'h et al. (2003) who noted that GRB hosts are significantly subluminous in the $K$-band (compare to their Fig. 5).

The estimated $R-K$ colour and the low redshift of the GRB 030329 host galaxy are consistent with the conclusions by Berger et al. (2003) and Tanvir et al. (2004), who found that the detected GRB hosts tend to be bluer and at lower redshifts than the submm-selected galaxies. In particular, it is illustrative of the position of the GRB 030329 host in the Fig. 6 colour-redshift diagram by Berger et al. (2003).

On the other hand, the estimated host galaxy stellar mass is lower than the ones calculated for the host sample of Chary et al. (2002), with the exception of the GRB 970508 host galaxy (Sokolov et al. 2001). 


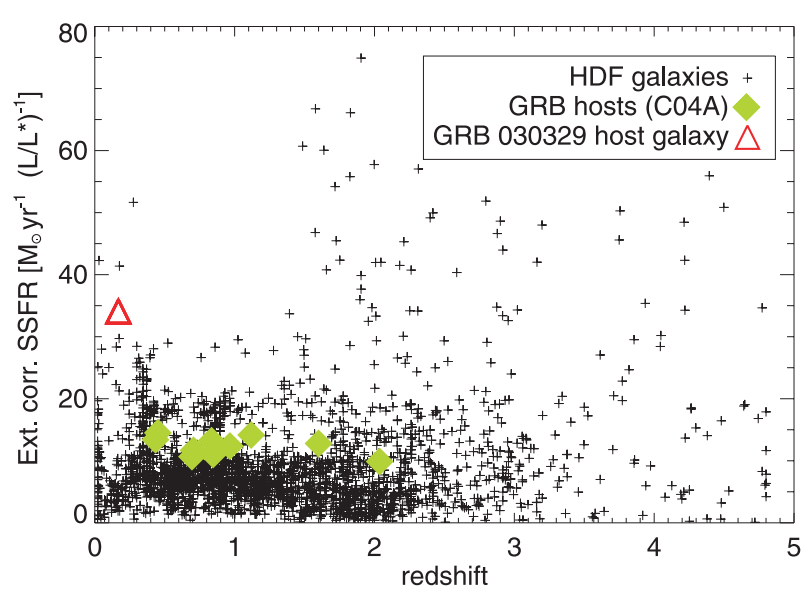

Fig. 4. The unextinguished SSFR of the C04A sample (filled diamonds) plus the GRB 030329 host galaxy (triangle) as a function of redshift. The crosses show the SSFR of the HDF when a S55 IMF is assumed. Note the high SSFR of the GRB 030329 host in comparison to the HDF galaxies at a similar redshift.

\section{Conclusions}

VLT spectroscopic observations show that the GRB 030329 host is a likely low metallicity galaxy, with $Z=0.004$ $\left(Z \sim Z_{\odot} / 5\right)$. From the SED fitting we infer that the host of GRB 030329 is most probably a starburst galaxy. This agrees with the C04A results, who found (based on an independent host sample) that GRB hosts correspond to Stb galaxies in $90 \%$ of the cases. We derived a dominant stellar population age of $150 \pm 80$ years, in agreement with the stellar age distribution of the C04A host galaxy sample (Mean age $=95 \pm 74 \mathrm{Myr}$ ). The low absolute magnitude of the host, $M_{B} \sim-16.5(L \sim$ $\left.0.016 L^{\star}\right)$, confirms its subluminous nature.

The SED fitting yields a consistent extinction value of $A_{\mathrm{v}}=0.6$ using either synthetic or empirical templates. This extinction is consistent with the mean extinction derived by C04A (median $A_{\mathrm{v}}=0.26_{-0.26}^{+0.51}$ ). The extinction derived from the Balmer emission lines is also consistent with the one derived from the SED.

We determine a consistent unextinguished SFR $0.6 M_{\odot} \mathrm{yr}^{-1}$ based on three independent diagnostic techniques: the $\mathrm{UV}$ continuum, the $[\mathrm{O} \mathrm{II}]$ and the Balmer emission lines. This consistency supports the inferred $A_{\mathrm{v}}=0.6$ value. The associated unextinguished SSFR $\left(\sim 34 M_{\odot} \mathrm{yr}^{-1}\left(L / L^{\star}\right)^{-1}\right)$ is higher than $\sim 93.5 \%$ of the SDSS galaxies at $z \sim 0.17$. Moreover, a statistical analysis carried out enhancing the C04A sample with the GRB 0303029 host confirms that GRB hosts shows higher SSFRs than HDF galaxies at similar redshifts. All these findings are consistent with the host galaxy being an active star forming galaxy.

We note the presence of a close galaxy with a similar redshift to the GRB host, which may be indicative of a possible clustering at $z \sim 0.17$. Multi-object spectroscopy of the GRB 030329 field may clarify this open issue.

Acknowledgements. J.G. acknowledges the support of a Ramón y Cajal Fellowship from the Spanish Ministry of Education and Science. The research of DPR has been supported by the Education Council of Junta de Andalucía, Spain. This research is partially supported by the Spanish Ministry of Science and Education through programmes ESP2002-04124-C03-01 and AYA2004-01515 (including FEDER funds). The observations presented in this paper were partially obtained under the ESO Programme 271.D-5006(A). P.J., G.J. and G.B. gratefully acknowledge support from a special grant from the Icelandic Research Council. V.V.S. and T.A.F. were supported by the Russian Foundation for Basic Research, grant No. 01-02-171061. We thank S. Savaglio for useful comments and C. Kehrig for helpful assistance analysing the VLT spectrum. We also acknowledge S. Toft, J.-M. Miralles, M. Bolzonella for their support with HyperZ and our anonymous referee for constructive comments.

\section{References}

Bell, E. F., McIntosh, D. H., Katz, N., \& Weinberg, M. D., 2003, ApJS, 149, 289

Berger, E., Cowie, L. L., Kulkarni, S.R., et al., 2003, ApJ, 588, 99

Binney, J., \& Merrifield, M., 1998, Galactic Astronomy, Princeton Series in Astrophysics

Bloom, J. S., Kulkarni, S. R., Djorgovski, S. G., et al. 1999, Nature, 401, 453

Bohlin, R. C., Colina, L., \& Finley, D. S. 1995, AJ, 110, 1316

Bolzonella, M., Miralles, J.-M., \& Pelló, R. 2000, A\&A, 363, 476

Bornancini, C. G., Martínez, H. J., Lambas, D. G., et al. 2004, ApJ, 614,84

Bruzual, A. G., \& Charlot, S. 1993, ApJ, 405, 538

Bruzual, A. G., \& Charlot, S. 2003, MNRAS, 344, 1000

Calzetti, D., Armus, L., Bohlin, R. C., et al. 2000, ApJ, 533, 682

Cardelli, J. A., Clayton, G. C., \& Mathis, J. S. 1989, ApJ, 345, 245

Castro-Tirado, A. J., \& Gorosabel, J. 1999, A\&AS, 138, 449

Chabrier, G. 2003, PASP, 115, 763.

Chary, R., Becklin, E. E., \& Armus, L. 2002, ApJ, 566, 229

Christensen, L., Hjorth, J., \& Gorosabel, J. 2004a, A\&A, 425, 913

Christensen, L., Hjorth, J., Gorosabel, J., et al. 2004b, A\&A, 413, 121

Cowie, L. L., Songaila, A., Hu, E. M., \& Cohen, J. G., 1996, AJ, 112, 839

Desai, V., Dalcanton, J. J., Mayer, L., et al. 2004, MNRAS, 351, 265.

Djorgovski, S. G., Frail, D. A., Kulkarni, S. R., et al. 2001a, ApJ, 562, 654

Djorgovski, S. G., Kulkarni, S. R., Bloom, J. S., et al. 2001b, in Gamma-ray Bursts in the Afterglow Era, ed. E. Costa, F. Frontera, \& J. Hjorth (Berlin Heidelberg: Springer), 218

Djorgovski, S. G., Bloom, J. S., Kulkarni, S. R., et al. 2003, ApJ, 591, L13

Eichler, D., Livio, M., Piran, T., \& Schramm, D. N. 1989, Nature, 340, 126

Fernández-Soto, A., Lanzetta, K. M., \& Yahil, A. 1999, ApJ, 513, 34

Fitzpatrick, E. L. 1986, AJ, 92, 1068

Fruchter, A., Levan A., Hook, R., et al. 2003, GCN Circ., 2243

Galama, T. J., Vreeswijk, P. M., van Paradijs, J., et al. 1998, Nature, 395,670

Gorosabel, J., Christensen, L., Hjorth, J., et al. 2003a, A\&A, 400, 127

Gorosabel, J., Klose, S., Christensen, L., et al. 2003b, A\&A, 409, 123

Greiner, J., Peimbert, M., Staban, C., et al. 2003, GCN Circ., 2020

Guziy, S., et al. 2005, A\&A, in preparation

Henden, A. A. 2003, GCN Circ., 2023

Hjorth, J., Thomsen, B., Nielsen, S. R., et al. 2002, ApJ, 576, 113

Hjorth, J., Sollerman, J., Møller, P., et al. 2003, Nature, 423, 847

Juneau, S., Glazebrook, K., Crampton, D., et al. 2005, ApJ, 619, L135

Kawabata, K. S., Deng, J., Wang, L., et al. 2003, ApJ, 593, L19

Kennicutt, R. C. 1998, ARA\&A, 36, 189

Kewley, L. J., \& Dopita, M. A. 2002, A\&A, 142, 35 
Kinney, A. L., Calzetti, D., Bohlin, R. C., et al. 1996, ApJ, 467, 38 Kouveliotou, C., Meegan, C. A., Fishman, G. J., et al. 1993, ApJ, 413, L101

Lee, H., Grebel, E. K., \& Hodge, P. W. 2003, A\&A, 401, 141

Le Floc'h, E., Duc, P.-A., Mirabel, I. F., et al. 2003, A\&A, 400, 499

Leitherer, C., Schaerer, D., Goldader, J. D., et al. 1999, ApJS, 123, 3

Lilly, S. J., Tresse, L., Hammer, F., Crampton, D., \& Le Fèvre, O. 1995, ApJ, 455, 108

MacFadyen, A. I., \& Woosley, S. E. 1999, ApJ, 524, 262

Matheson, T., Garnavich, P. M., Stanek, K. Z., et al. 2003, ApJ, 599, 394

Narayan, R., Paczyńsky, B., \& Piran, T. 1992, ApJ, 395, L83

Oke, J.B. 1990, AJ, 99, 1621

Osterbrock, D. E., 1989, Astrophysics of Gaseous Nebulae and Active Galactic Nuclei (Mill Valley: University Science Books)

Paczyński, B. 1998, ApJ, 494, L45

Peacock, J. A. 1983, MNRAS, 202, 615

Pei, Y. C. 1992, ApJ, 395, 130

Portinari, L., Chiosi, C., \& Bressan, A. 1998, A\&A, 334, 505

Prévot, M. L., Lequeux, J., Prévot, L., Maurice, E., \& Rocca-Volmerange, B. 1984, A\&A, 132, 389

Price, P. A., Fox, D. W., Kulkarni, S. R., et al. 2003, Nature, 423, 844

Prochaska, J. X., Bloom, J. S., Chen, H.-W., et al. 2004, ApJ, 611, 200
Reif, K., Poschmann. H., Bagschik. K., et al. 2000, in Optical Detectors for Astronomy, ed. P. Amico and J. W. Beletic (Kluwer Academic Publisher), 143

Ricker, G. R., et al. 2003, IAU Circ., 8101

Salpeter, E. E. 1955, ApJ, 121, 161

Schechter, P. 1976, ApJ, 203, 297

Schlegel, D. J., Finkbeiner, D. P., \& Davis, M. 1998, ApJ, 500, 525

Seaton, M. J. 1979, MNRAS, 187, 73

Sokolov, V. V., Fatkhullin, T. A., Castro-Tirado, A. J., et al. 2001, A\&A, 372, 438

Sokolov, V. V., Fatkhullin, T. A., Komarova, V. N., et al. 2003, Bulletin of the Special Astrophysical Observatory RAS, 56, 5

Sollerman, J., Östlin, G., Fynbo, J., et al. 2005, New Astron., in press [arXiv: astro-ph/0506686]

Stanek, K. Z., Matheson, T., Garnavich, P. M., et al. 2003, ApJ, 591, L17

Tanvir, N. R., Barnard, V. E., Blain, A. W., et al. 2004, MNRAS, 352, 1073

York, D. G., Adelman, J., Anderson, J. E., et al. 2000, AJ, 120, 1579

Vanderspek, R., Crew, G., Doty, J., et al. 2003, GCN Circ., 1997

Vanzella, E., Cristiani, S., Saracco, P., et al. 2001, AJ, 122, 2190

Williams, R. E., Baum, S., Bergeron, L. E., et al. 2000, AJ, 120, 2735

Woosley, S. E. 1993, ApJ, 405, 273 